\title{
Translation, cultural adaptation and validation of a Spanish version of the Irritable Bowel Syndrome Severity Score
}

\author{
Cristina Almansa ${ }^{1,2}$, Raquel García-Sanchez ${ }^{1}$, Marta Barceló ${ }^{1}$, Manuel Díaz-Rubio ${ }^{1}$ and Enrique Rey ${ }^{1}$ \\ ${ }^{1}$ Unit of Functional Digestive Disorder. Department of Gastroenterology. Hospital Clínico San Carlos. School of \\ Medicine. Universidad Complutense. Instituto de Investigación Sanitaria. Hospital Clínico San Carlos (IdISSC). Madrid, \\ Spain. ${ }^{2}$ Division of Gastroenterology. Mayo Clinic Jacksonville. Florida, USA
}

\begin{abstract}
Background: the Irritable Bowel Syndrome Severity Score (IBSSS) is a questionnaire only available in English that classifies IBS patients according to the severity of their symptoms and can be used to guide and monitor the treatment.

Aims: to adapt and validate into Spanish the English version of the IBSSS questionnaire.

Methods: the Spanish version of the questionnaire was obtained through a process of translation-evaluation of the comprehensibility and back translation. In a later phase we evaluated the applicability $(\mathrm{n}=15)$, reproducibility $(\mathrm{n}=31)$ and sensitivity to change $(n=40)$ of the Spanish version of the questionnaire. Finally we evaluated an alternative version of the ISBSS using a numerical scoring system instead of the original analog visual scale $(n=40)$.

Results: the Spanish version of the IBSSS showed an excellent reproducibility $(r=0.81$ for global score) and an adequate sensitivity to change: a decrease of 45 points or more identified worsening of IBS with a $70.6 \%$ sensitivity and $87.5 \%$ specificity; an increase of 45 points or more identified improvement of IBS with a $85.7 \%$ sensitivity and $87.5 \%$ specificity. The severity score was practically the same regardless of the scoring system used $(r=0.96)$.

Conclusions: the Spanish version of the IBSSS is a reproducible tool that is able to identify relevant changes over the course of the disease. The use of a numerical scoring system is a valid alternative to the visual scale that improves the applicability of the questionnaire to situations when the written communication is limited or not possible.
\end{abstract}

Key words: Irritable bowel syndrome. Severity essessment. Questionnaire. Acknowledgement: This study has been supported with an unrestricted grant
from the Fundacion Mutua Madrileña (FMM).

Received: 05-07-11.

Accepted: 08-07-11.

Correspondence: Enrique Rey. Department of Gastroenterology. Hospital Clinico Universitario San Carlos. C/ Profesor Martin Lagos, s/n. 28040 Madrid, Spain.

e-mail: rey.enrique@med.ucm.es
Almansa C, García-Sanchez R, Barceló M, Díaz-Rubio M, Rey E. Translation, cultural adaptation and validation of a Spanish version of the Irritable Bowel Syndrome Severity Score. Rev Esp Enferm Dig 2011; 103: 612-618.

\section{BACKGROUND}

Irritable bowel syndrome (IBS) is the most common and representative of all functional gastrointestinal disorders (1). In Spain, IBS prevalence ranges between 3 and 12\% depending on the diagnostic criteria used for its definition (2). IBS is a common cause of medical consultation, representing up to $3 \%$ of all patients seen in primary care and $25 \%$ of those attended in gastroenterology (3).

Though, a previous survey did not show relevant differences between primary care doctors and gastroenterologists in the overall management of IBS (4), not all patients require the same type of care. In general, it is assumed that IBS patients with mild symptoms can be perfectly managed in primary care while those with severe forms will benefit from specialized attention (5). Overall, in Spain diagnostic and treatment strategies are usually based on the patients' symptoms (6), however patients with IBS require more individualized treatment. Current guidelines recommend specific therapies not only based on the predominant symptoms but also according symptoms' severity (7). To date, there are a scarce number of standardized tools that can be used as objective measurement of IBS severity (8).

In 1997 British researchers led by Peter Whorwell designed a simple scoring system (Irritable Bowel Syndrome Severity Score, IBSSS) that classified IBS patients according the severity of their symptoms and that would be used as guidance when monitoring treatment outcomes in both clinical and research settings (9). The IBSSS has clear advantages over other severity questionnaires $(10,11)$ and therefore it has been extensively used in different clin- 
ical trials. Nonetheless, an adapted and validated version of this questionnaire is not available in Spanish yet.

Applicability is one of the main disadvantages of this instrument (10). Visual analog scales, as those included in the IBSSS, are not applicable to patients with visual and/or motor disabilities; may be difficult to understand by older people, not suitable for phone interviews and require an specific software package when used in internet based surveys (12-14). However, these limitations might be avoided by using numerical scales, which are easy to verbalize and suitable for phone and web based surveys.

The aims of this study are first to adapt and validate into Spanish the IBSSS questionnaire, and second, to evaluate an alternative version of this instrument using a numerical scoring system applicable to people with disabilities and suitable for its use in phone and web based surveys.

\section{METHOD}

The Irritable Bowel Syndrome Severity Score (IBSSS) was developed and validated by Francis et al. in Manchester in 1997 (9). This is a 5-item instrument that measures intensity (visual analog scale) and frequency (number of days) of abdominal pain; intensity of abdominal distension (visual analog scale); bowel movements satisfaction (visual analog scale) and potential impact of IBS on the patient's daily life (visual analog sale), all of them referred to the last 10 days.

Each item can be scored from 0-100 (according to the value marked by the patient on the visual analog scale or the number of days multiplied by 10), thus the overall IBS severity score ranges from 0 to 500; which according to the original validation (9) can be classified as: $<75$ healthy subjects or disease in remission; 75-175 mild disease; 175300 moderate and $>300$ severe disease.

\section{Translation}

The original questionnaire was independently translated into Spanish by 2 native Spanish gastroenterologists (CA, ER). Later, these two independent translations were unified in one single consensual translation (first draft).

\section{Evaluation of the comprehensibility}

The first draft of the questionnaire was administered to a small sample $(n=15)$ of patients attending the Functional Digestive Disorders Unit at the Hospital Clínico San Carlos, Madrid. Participants were mainly recruited among those with the lowest education level in order to warranty the comprehensibility of the questionnaire by all type of subjects. Participants self completed the survey and later discussed its comprehensibility with the researchers, who added some final changes to the questionnaire based on patient's feedback (second draft).

\section{Back translation into English}

Back translation of the Spanish version into English was performed by an official native English translator. The original IBSSS was unbeknownst to the translator. Finally, both researchers and translator compared the original English questionnaire with the back translation and draw up a consensual final version into Spanish.

\section{Applicability}

Fifteen IBS patients fulfilling Rome III criteria filled the questionnaire; of those 5 were recruited from primary care, 5 from the community gastroenterology offices and 5 from the Functional Digestive Disorder Unit in our Hospital. These patients were classified as presenting mild, moderate and severe IBS according to the level of specialized care that they required. Patients' ability to understand and respond the questionnaire was assessed in this phase.

\section{Reproducibility}

Fifty Rome III IBS patients attending the Functional Digestive Disorders Unit with stable symptoms according to the judgment of one of the researchers (ER), were invited to fill the questionnaire and then asked to return 7-15 days later to fill the questionnaire again. None of the patients received any treatment during that interval of time. Reproducibility was assessed by comparing the responses to both questionnaires by Pearson correlation.

\section{Sensitivity to change}

A further group of 40 Rome III IBS patients attending our unit completed the IBSSS questionnaire and were invited to fill it again in a follow up visit. In this interval of time patients received adequate treatment if required. An additional question was added to the questionnaire in the second visit: "compared with the previous visit, how do you feel now about your IBS symptoms?" The possible responses were: same, better or worse. We compared the IBSSS scores (total and by individual items) between both visits and analyzed if there were differences among groups (those who were feeling better, same or worse) using an ANOVA test and Bonferroni correction. ROC curve analysis was used to compare among those that were better $v s$. same $v s$. worse.

\section{Assessment of the numerical scoring system}

We developed a modified version of the IBSSS that included a numerical scoring system instead of the VAS. The numerical scale ranged from 0 (nothing) to 12 (the maximum level, totally). We asked 40 patients to complete 


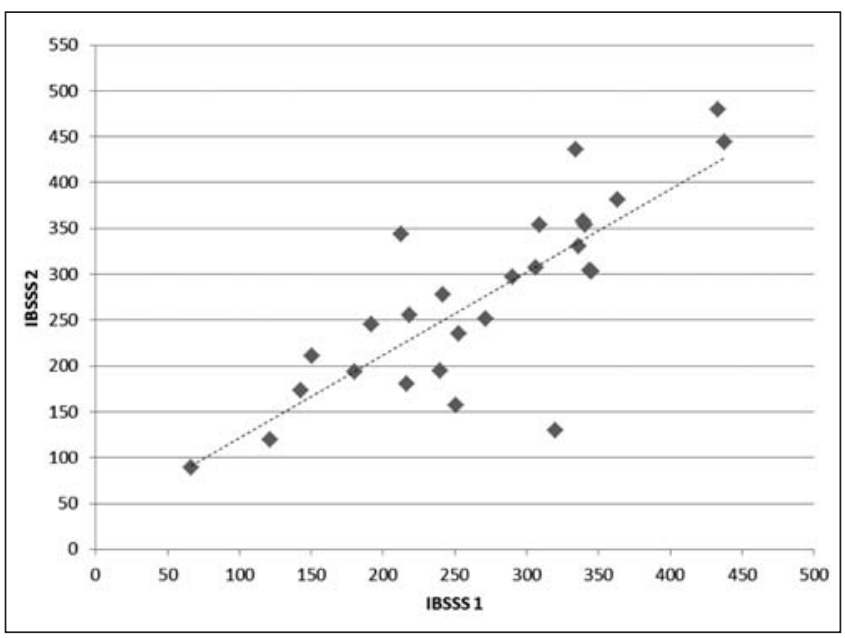

Fig. 1. Graphic representation of the IBSSS reproducibility (Pearson correlation).

both versions of the questionnaire in the same visit; the order how the questionnaires were administered changed between patients, thus half of the patients responded first the VAS version (at the beginning of the appointment) and later (at the end of the appointment) the numerical version and vice versa. Responses to both questionnaires were compared by Pearson correlation.

\section{Statistical analysis}

The statistical analysis previously described was performed using SPSSS for Windows v.15.0 (Chicago, IL).

\section{Ethical issues}

This study was approved by the Hospital Clínico San Carlos Ethics Committee and all data were managed con-

\section{Table I. Correlation coefficients for the reproducibility of global and individual scores of the IBSSS (before and after)}

\begin{tabular}{llll}
\hline & $\begin{array}{l}\text { Baseline score } \\
\text { (range) }\end{array}$ & $\begin{array}{l}\text { Correlation } \\
\text { coefficient (r) }\end{array}$ & $p$ value \\
\hline $\begin{array}{l}\text { Abdominal pain severity } \\
\text { (0-100) }\end{array}$ & $0-98$ & 0.67 & $<0.0001$ \\
$\begin{array}{l}\text { Abdominal pain duration } \\
\text { (days) }\end{array}$ & $0-10$ & 0.79 & $<0.0001$ \\
$\begin{array}{l}\text { Abdominal distension } \\
\text { severity (0-100) }\end{array}$ & $0-100$ & 0.80 & $<0.0001$ \\
$\begin{array}{l}\text { Bowel habit satisfaction } \\
\text { (0-100) }\end{array}$ & $0-100$ & 0.45 & 0.01 \\
$\begin{array}{l}\text { Life disruption (0-100) } \\
\text { IBSSS }\end{array}$ & $0-100$ & 0.82 & $<0.0001$ \\
\hline & $68-438$ & 0.81 & $<0.0001$ \\
\hline
\end{tabular}

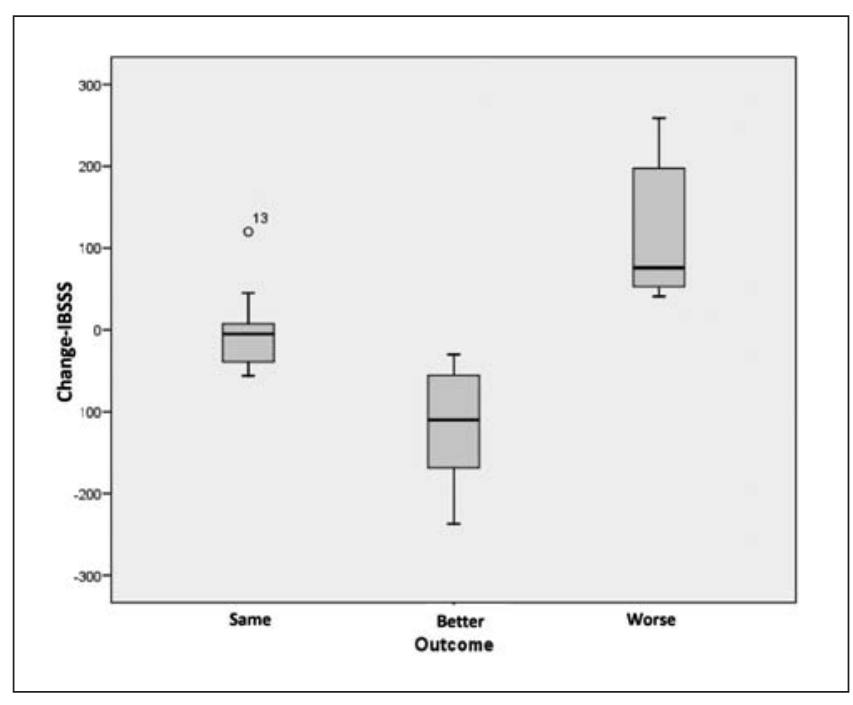

Fig. 2. Changes in the IBSSS scores according to the patient's self-assessment (same, better, worse).

fidentially by the researchers according to the Spanish regulation for personal data protection (law 15/99).

\section{RESULTS}

\section{Comprehensibility and applicability}

After reviewing and discussing the first draft of the questionnaire with the patients we noticed that 3 of 15 misunderstood the bowel habit satisfaction assessment and responded this question backwards. Thus, we decided to change the order of the scale, $0 \%$ (very happy) would be $0 \%$ (very unhappy) and $100 \%$ (very unhappy) would be substituted by $100 \%$ (very happy). Following the back translation process, we asked other 15 patients to fill the new version. All participants in this second round understood and responded adequately to all the questions. The time needed to fill the questionnaire was variable between 3-10 minutes depending on the patients' level of literacy.

\section{Reproducibility}

Thirty-one of 50 patients completed the reproducibility study within 6-19 days of interval. Twenty-three of 31 $(98 \%)$ were females, mean age $41.7 \pm 14.2$ (range 20-79). The global scoring in the group ranged between 66 and 437. According to the severity scores in the original IBSSS questionnaire 1 patient was in remission, 3 had mild disease, 13 moderate and 14 severe IBS.

Comparison of the global score showed an excellent correlation, $\mathrm{r}=0.81$ (Fig. 1); a good correlation was also found for each of the 5 individual items that constitute the questionnaire, as it is shown in table I. 
Table II. Average changes in the IBSSS scores $(95 \% \mathrm{Cl})$ based on the patient's self-assessment

\begin{tabular}{llll}
\hline & Same $(n=16)$ & Better $(n=17)$ & Worse $(n=7)$ \\
\hline IBSSS & $-2.8(-25.8 ; 20.3)$ & $-108.9(-144.1 ;-73.8)^{\mathrm{a}}$ & $125.3(35.9 ; 214.6)^{\mathrm{a}, \mathrm{b}}$ \\
Abdominal pain severity (VAS) & $-4.1(-13.7 ; 5.4)$ & $-20.2(-31.5 ;-8.8)$ & $26.3(8.7 ; 43.9)^{\mathrm{a}, \mathrm{b}}$ \\
Abdominal pain duration (days) & $-0.1(-1.3 ; 1.1)$ & $-3.5(-5.2 ;-1.9)^{\mathrm{a}}$ & $4.9(1.4 ; 8.3)^{\mathrm{a}, \mathrm{b}}$ \\
Abdominal distension severity & $0.5(-7.6 ; 8.5)$ & $-13.4(-23.5 ;-3.3)$ & $24.0(3.0 ; 45.1)^{\mathrm{a}, \mathrm{b}}$ \\
Bowel habit satisfaction & $3.1(-10.1 ; 16.3)$ & $24.6(9.3 ; 39.9)$ & $-2.1(-18.6 ; 14.4)$ \\
Life disruption & $5.0(-9.2 ; 19.2)$ & $-16.7(-25.5 ;-7.8)$ & $24.2(-9.8 ; 58.2)^{\mathrm{b}}$ \\
\end{tabular}

"p $<0.05$ compared with "same" in post hoc analysis; " $\mathrm{p}<0,05$ compared with "better" in post hoc analysis.

\section{Sensitivity to change}

Forty patients responded both questionnaires within 1 and 9 months. Of those, 29 were females $(72.5 \%)$, mean age $38.9 \pm 13$ years (range 22-69). IBSSS basal scores ranged between 126 (mild disease) and 412 (severe disease). Patients were classified at base line, according to the original questionnaire's scores as presenting mild $(n=9)$, moderate $(n=16)$ and severe $(n=15)$ disease.

As expected, comparisons of the IBSSS results obtained before and after the intervention showed low correlation ( $\mathrm{r}$ $=0.36$ ). The scores clearly differed among patients according to their self-assessment, with significantly different results between those classified as same $v s$. better, same $v s$. worse and better $v s$. worse.

We calculated the score variation required to predict the result "better" (compared with "same") by ROC analysis (area under the curve 0.93); and estimated that a decrease of 45 points in the IBSSS score predicted this result with a sensitivity of $70.6 \%$ and a specificity of $87.5 \%$. The area under the curve obtained to predict the result "worse" (compared to "same") was of 0.95 ; increases in the IBS score

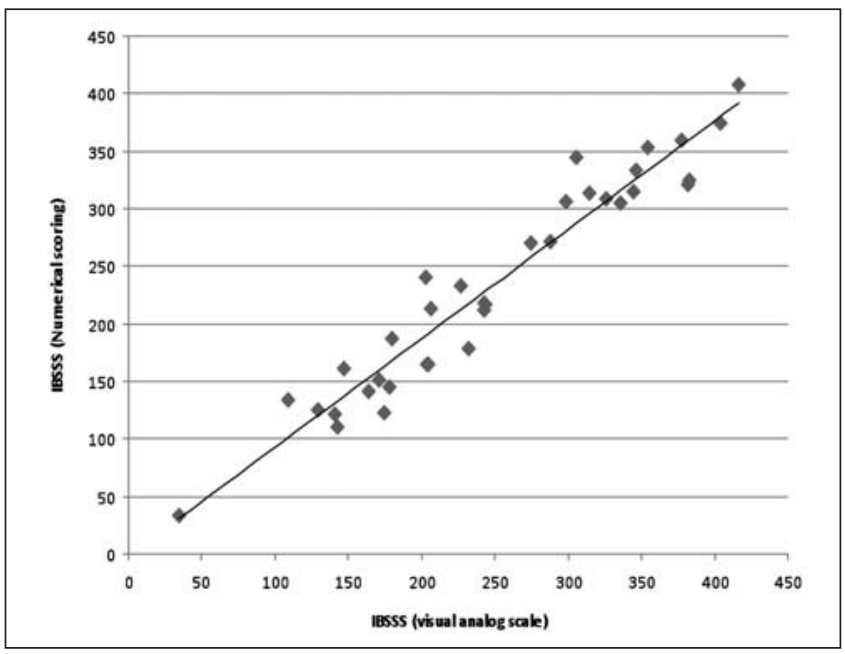

Fig. 3. Comparison of IBSSS results: visual analog scale vs. numerical scoring system. of 45 points will show this result with a sensitivity of $85.7 \%$ and a specificity of $85.7 \%$.

\section{Assessment of the numerical scoring system}

Forty patients filled the two different versions of the IBSSS questionnaire, one using a VAS and the other one using a numerical scoring system. Thirty-four (85\%) of the participants were females, mean age $40.2 \pm 12.7$ years (range 22-64). IBSSS basal scores ranged between 34 (disease in remission) and 416 (severe disease). At base line, patients' severity was classified as "in remission" $(\mathrm{n}=1)$, "mild" $(n=9)$, "moderate" $(n=16)$ and "severe" $(n=14)$.

Comparison of global scores obtained through both types of rating scales showed excellent correlation, $r=0.96$ (Fig. 3 ), as well as a good level of correlation for the 5 individual items that constitute the IBSSS, as shown in table III.

\section{DISCUSSION}

These results suggest the adequate reproducibility and sensitivity to change of the Spanish version of the IBSSS. In addition, the modified version using a numerical system instead of the analogue visual scale seems to be a valid alternative, as the IBSSS severity score remains unchanged regardless of the scoring system used.

Table III. Correlation coefficients for the global score and individual items of the IBSSS using both rating scales (VAS vs. numerical)

\begin{tabular}{lll}
\hline & $\begin{array}{l}\text { Correlation } \\
\text { coefficient }(r)\end{array}$ & $p$ value \\
\hline Abdominal pain severity & 0.87 & $<0.0001$ \\
Abdominal pain duration (days) & 0.98 & $<0.0001$ \\
Abdominal distension severity & 0.90 & $<0.0001$ \\
Bowel habit satisfaction & 0.89 & $<0.0001$ \\
Life disruption & 0.85 & $<0.0001$ \\
IBSSS & 0.97 & $<0.0001$
\end{tabular}


The translation and validation process of the IBSSS was performed according current methodology recommendations (15). Asking the right question using the most appropriate wording is of paramount importance when designing a new questionnaire or adapting a previous one. Therefore it seems obvious that a simple translation-back translation does not warranty the validity of the resulting questionnaire that will require of specific cultural validation (15). For instance, when adapting the IBSSS we had to modify the response to the bowel habit satisfaction assessment; which was originally rated from $0 \%$ (very happy) to $100 \%$ (very unhappy). It seems as in Spanish that response was senseless, which was noted when we found that most of the patients complaining of severe diarrhea or constipation were paradoxically rating this item as if they were very happy or satisfied. Once the rating scale was changed the problem was solved.

As it was reflected by the adequate correlation between repeated scores of global and individual symptom's severity the Spanish version of the IBSSS presents an excellent reproducibility. Bowel habit satisfaction seems to be the least reproducible of all features; that could be due to the fact that bowel habit is not a single item and comprises multiple characteristics (frequency, consistency, urgency, straining, and feeling of incomplete evacuation) suitable of changing over time.

From a clinical perspective, one of the most useful findings in this study is that the Spanish version of the IBSSS is highly sensitive to change. It is remarkable that in both validations of the IBSSS, the original in English and the Spanish validation, a change of 50 was considered clinically significant and indicative of improvement. It is noteworthy that diagnostic threshold in both validations agree despite methodological differences. Indeed, while in the original the existence of potential clinical changes was judged by a physician, we asked the patients to self assess their clinical course. It is also of relevance, that the global score seems to be better than each of the individual items' scores, which suggests that the severity of IBS does not rely exclusively in the characteristics of abdominal pain, as it was measured by other severity scoring scales such as the "functional bowel disorder severity index" (FBDSI) (16).

It is noticeable that the results obtained with the numerical scoring show an excellent correlation with those obtained by using the VAS, not only for the IBSSS global scores but also for the scores of each of the individual items assessed in the questionnaire; which implies that this instrument can be adaptable to a broad range of situations.

In summary, the Spanish version of the IBSSS seems to be a reproducible tool that is able to identify relevant changes over the course of the disease. The use of a numerical scoring system is a valid alternative to the visual scale that improves the applicability of the questionnaire to situations when the written communication is limited or not possible.

\section{REFERENCES}

1. Rey E, Talley NJ. Irritable bowel syndrome: novel views on the epidemiology and potential risk factors. Dig Liver Dis 2009;41:772-80.

2. Mearin F, Badia X, Balboa A, Baro E, Caldwell E, Cucala M, et al Irritable bowel syndrome prevalence varies enormously depending on the employed diagnostic criteria: comparisons of Rome II versus previous criteria in a general population. Scand J Gastroenterol 2001; 36:1155-61.

3. Thompson WG, Heaton KW, Smyth GT, Smyth C. Irritable bowel syndrome in general practice: Prevalence, characteristics, and referral. Gut 2000;46:78-82.

4. Almansa C, Rey E, Bolaños E, Palma M, Alvarez Sánchez A, DíazRubio M. Spanish physicians point of view on irritable bowel syndrome (IBS). Results of a Delphi survey. Rev Esp Enferm Dig 2007;99:2107 .

5. Drossman DA, Thompson WG. The irritable bowel syndrome: review and a graduated multicomponent treatment approach. Ann Intern Med 1992;116:1009-16.

6. Almansa C, Diaz-Rubio M, Rey E. The burden of management of patients with IBS: results from a survey in Spanish gastroenterologists. Rev Esp Enferm Dig 2011;103:570-5.

7. Drossman DA, Camilleri M, Mayer EA, Whitehead WE. AGA technical review on irritable bowel syndrome. Gastroenterology 2002;123:2108-31.

8. Lembo A, Ameen VZ, Drossman DA. Irritable bowel syndrome: toward an understanding of severity. Clin Gastroenterol Hepatol 2005;3:717-25.

9. Francis CY, Morris J, Whorwell PJ. The irritable bowel severity scoring system: a simple method of monitoring irritable bowel syndrome and its progress. Aliment Pharmacol Ther 1997;11:395-402.

10. Spiegel B, Camilleri M, Bolus R, Andresen V, Chey WD, Fehnel S, et al. Psychometric evaluation of patient-reported outcomes in irritable bowel syndrome randomized controlled trials: a Rome Foundation report. Gastroenterology 2009;137:1944-53.e1-3.

11. Spiegel BM, Bolus R, Agarwal N, Sayuk G, Harris LA, Lucak S, et al. Measuring symptoms in the irritable bowel syndrome: development of a framework for clinical trials. Aliment Pharmacol Ther 2010;32:127591.

12. Flaherty SA. Pain measurement tools for clinical practice and research. AANAJ 1996;64:133-40.

13. Gagliese L, Weizblit N, Ellis W, Chan VW. The measurement of postoperative pain: a comparison of intensity scales in younger and older surgical patients. Pain 2005;117:412-20.

14. Marsh-Richard DM, Hatzis ES, Mathias CW, Venditti N, Dougherty DM. Adaptive Visual Analog Scales (AVAS): a modifiable software program for the creation, administration, and scoring of visual analog scales. Behav Res Methods 2009;41:99-106.

15. Sperber AD. Translation and validation of study instruments for crosscultural research. Gastroenterology 2004;126:S124-S128.

16. Drossman DA, Li Z, Toner BB, Diamant NE, Creed FH, Thompson $\mathrm{D}$, et al. Functional bowel disorders. A multicenter comparison of health status and development of illness severity index. Dig Dis Sci 1995; 40:986-95 